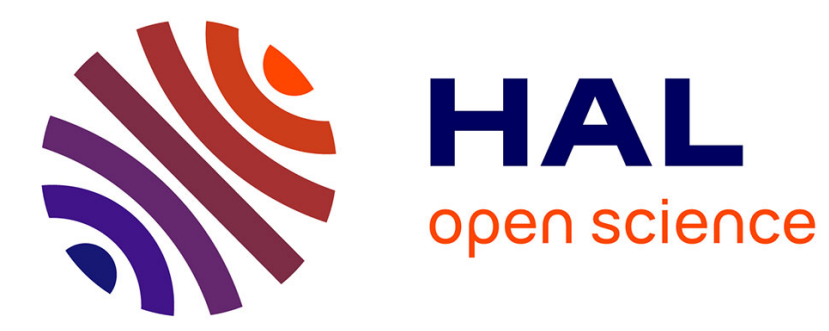

\title{
Un aspect négligé par l'étude de l'engagement sportif: la multipratique et ses modalités
}

Muriel Augustini, Paul Irlinger, Catherine Louveau

\section{To cite this version:}

Muriel Augustini, Paul Irlinger, Catherine Louveau. Un aspect négligé par l'étude de l'engagement sportif: la multipratique et ses modalités. Loisir et Société / Society and Leisure, 1996, 19 (1), pp.237-262. hal-01825922

\section{HAL Id: hal-01825922 \\ https: / hal-insep.archives-ouvertes.fr/hal-01825922}

Submitted on 28 Jun 2018

HAL is a multi-disciplinary open access archive for the deposit and dissemination of scientific research documents, whether they are published or not. The documents may come from teaching and research institutions in France or abroad, or from public or private research centers.
L'archive ouverte pluridisciplinaire HAL, est destinée au dépôt et à la diffusion de documents scientifiques de niveau recherche, publiés ou non, émanant des établissements d'enseignement et de recherche français ou étrangers, des laboratoires publics ou privés. 


\title{
Un aspect négligé par l'étude de l'engagement sportif: la multipratique et ses modalités
}

\author{
Muriel AUGUSTINI, Paul IRLINGER, Catherine LOUVEAU
}

INSEP, Département de la recherche, Paris, France

Article publié dans : Loisir et société (ISSN: 0705-3436), 1996, vol. 19, $n^{\circ} 1$, pp. 237-262

\section{RÉSUMÉ}

Le nombre d'activités pratiquées par la même personne est une variable généralement négligée dans l'étude des pratiques sportives ; ce texte lui est entièrement consacré. La multipratique, opposée à la monopratique, est étudiée dans ses modalités et leurs variations dans différents secteurs spatiotemporels de pratique (en vacances, durant le reste de l'année à l'intérieur et hors du domicile) en fonction des principales caractéristiques socio-démographiques (âge, sexe, catégorie socioprofessionnelle) et selon la nature institutionnelle de l'engagement sportif (pratique informelle, pratique dans le cadre des fédérations sportives et pratique de compétition). L'analyse de données d'enquête aboutit aux résultats suivants : (1) L'inégalité de la distribution sociale est encore plus accentuée pour la multipratique que pour la pratique sportive ; (2) La participation aux compétitions, même de haut niveau, ne se fait pas, comme on aurait pu le supposer, au détriment du nombre de sports pratiqués. Bien au contraire, ce sont les compétiteurs de haut niveau qui présentent la multipratique la plus riche.

Lorsqu'il s'agit d'estimer la « sportivité » d'une population, le premier critère pris en considération est sa proportion de pratiquants : on dira ainsi que tel pourcentage d'une population pratique du sport. Ce taux, généralement déterminé à l'aide d'enquêtes par questionnaire réalisées sur des échantillons représentatifs, constitue certes une donnée indispensable pour décrire la pénétration d'une population par les activités physiques et sportives (APS), mais il ne fournit qu'une information élémentaire et trop globale. Le plus souvent une formulation plus rigoureuse conduirait à préciser que durant telle période de référence, tel pourcentage de la population considérée a pratiqué au moins une fois une APS. Lorsque la période de référence explorée par l'enquête correspond à une année, ce qui est le cas le plus courant, le seuil séparant les non-pratiquants des pratiquants s'avère très peu exigeant, a fortiori si la définition du sport qui sous-tend l'enquête est elle-même très ouverte et englobe non seulement les sports proprement dits, mais aussi toutes les APS d'exercice, d'agrément et d'hygiène. Sur de telles bases, l'enquête sur « Les pratiques sportives des Français » ${ }^{1}$ a montré que 73,8 \% des Français âgés de 12 à 74 ans « font du sport $»^{2}$.

Afin de dépasser ce niveau élémentaire, nous avons, pour rendre compte de la sportivité de la population, utilisé d'autres caractéristiques telles, par exemple, la nature des APS pratiquées, la fréquence de la pratique, son inscription institutionnelle, etc. Parmi les dimensions de la pratique permettant de préciser cette description et d'affiner l'analyse, nous nous sommes attachés à l'étude approfondie des modalités et variations de la multipratique. Cette analyse détaillée est réalisée en utilisant comme données certains résultats de l'enquête sur les pratiques sportives des Français. Seules les personnes ayant déclaré au moins une APS $(N=2342)$ ont été retenues pour cette exploitation secondaire.

Au-delà des proportions respectives de « mono- » et de « multipratiquants» ainsi que du nombre d'APS pratiquées, nous avons étudié les variations de cette grandeur selon les principales variables sociodémographiques ; nous avons examiné la distribution de cette grandeur sur les secteurs spatiotemporels distinguant les pratiques de vacances de celles du reste de l'année, et les exercices accomplis au domicile des sports pratiqués à l'extérieur. Nous avons analysé enfin les modulations de la multipratique selon le degré d'engagement institutionnel, et notamment compétitif du pratiquant. 
L'étude approfondie de la multipratique et des ses modalités se trouve en quelque sorte appelée par l'évolution récente du phénomène sportif. Les années soixante et soixante-dix ont été marquées dans les pays occidentaux par l'accroissement quantitatif souvent spectaculaire de la population des pratiquants. L'attention des media et celle des responsables politiques du sport avaient tendance, de ce fait, à se focaliser sur l'évolution des taux de pratique. Bien des études impulsées par ces instances portaient ainsi sur les groupes sociaux à faible participation sportive (femmes, personnes âgées et minorités ethniques). Les campagnes de promotion dites " sport pour tous» caractérisaient cette période d'expansion d'un sport conçu comme facteur de la santé et du bien-être publics.

$\mathrm{Au}$ début des années quatre-vingts, la montée des taux de pratique s'est poursuivie, nourrie essentiellement par la croissance de la participation des femmes et le prolongement des APS de plus en plus tard dans le cycle de vie. Les années suivantes ont vu les taux moyens se stabiliser et même régresser, selon certains auteurs (Kamphorst \& Roberts, 1989) dans quelques pays européens. Dès lors, ces taux généraux ont perdu de leur intérêt, l'étude statistique du développement et de l'évolution du phénomène sportif devant recourir à des indicateurs plus fins et plus complexes. De ce point de vue, l'analyse de la multipratique présente des ressources fortement négligées à ce jour.

Il est vraisemblable que la multipratique et ses modalités manifestent, dans le domaine sportif, certaines grandes tendances à l'oeuvre dans les sociétés contemporaines (Clément, Defrance \& Pociello, 1994): la multiplication et la labilité croissante des appartenances groupales ; la transformation des adhésions affinitaires en rapports de consommation de services; et l'ouverture de l'éventail des pratiques culturelles comme marqueur de la position sociale.

\section{Monopratique, multipratique : les données générales}

Quand ils s'adonnent à une activité physique ou sportive, les Français sont dans leur grande majorité multipratiquants : plus des trois quarts d'entre eux sont dans ce cas, à peine plus de $22 \%$ s'adonnant à une seule activité, le plus souvent durant l'année en dehors du domicile. Ces résultats sont très proches de ceux de Roberts et al. (1991) pour le Royaume-Uni : parmi les 1387 adultes interrogés sur leur carrière sportive, $72 \%$ pratiquaient simultanément au moins deux APS durant l'année.

Ces données générales varient plus ou moins sensiblement selon les caractéristiques sociodémographiques des individus. Si le sexe influence peu l'engagement dans une multipratique ${ }^{3}$, en revanche, l'âge s'avère, ainsi qu'on pouvait s'y attendre, porteur d'une forte différenciation (Rudman, 1989) : des plus jeunes aux plus âgés, la monopratique devient environ 4 fois plus fréquente (Tableau 1). L'âge, qui renvoie entre autres à sa dimension biologique, est une donnée valant pour les hommes comme pour les femmes : pour tous, la multipratique devient nettement moins fréquente au-delà de 50 ans. De même est-elle, toutes proportions gardées, tout à fait comparable en deçà de 25 ans.

Restent les 25-49 ans et en particulier les 35-49 ans, tranches d'âge où les différences entre hommes et femmes sont assez fortement accusées, ces dernières étant bien plus fréquemment monopratiquantes que leurs homologues masculins. À une période de leur vie, les femmes seraient donc amenées à interrompre ou à réduire le nombre de leurs engagements, dans des pratiques physiques tout au moins. On est évidemment tenté d'invoquer ici le cumul des tâches valant pour nombre de femmes ainsi que l'éventuelle présence de jeunes enfants, bref de recourir à la question du temps, dans ses aspects quantitatifs et qualitatifs. Or, cette dimension garde toute sa complexité ${ }^{4}$ et il demeure toujours difficile d'établir, en ce domaine, des relations simples de causes à effets.

Les femmes se marient aujourd'hui relativement tard, à 26 ans en moyenne ; en outre, nombre d'entre elles ont leur premier enfant au-delà de cet âge ${ }^{5}$. Entre 25 et 54 ans, $72 \%$ exercent une activité professionnelle, situation que connaissent $68 \%$ des mères de famille ayant 1 ou 2 enfants. La combinaison de ces différents éléments tiendrait donc comme explication... si les femmes au foyer (qui disposent toujours, ainsi qu'on le sait, de plus de temps libre que les autres femmes, qu'elles aient ou non des enfants) étaient plus fréquemment multipratiquantes que les femmes actives, et si la présence et le nombre d'enfants pesaient sur le fait d'avoir une mono ou multipratique physique ou sportive ${ }^{6}$. Or, les résultats ne le confirment pas, bien au contraire : 
- Le taux de monopratique est plus élevé parmi les femmes au foyer $(31,8 \%)$ que parmi les femmes cadres ou membres des professions intellectuelles supérieures $(10,8 \%)$, membres des professions intermédiaires $(24,1 \%)$ et encore employées $(25,7 \%)$; on peut donc être une femme active, ne pas appartenir aux catégories sociales les plus favorisées et cependant s'adonner à une multipratique physique ou sportive (Tableau 2).

- Les fluctuations observables dans les modalités de pratique ne semblent pas résulter du nombre d'enfants vivant au foyer : aucune tendance ne révèle que la multipratique serait moins probable quand augmente le nombre d'enfants. Les hommes ayant deux enfants sont même plus nombreux à être multipratiquants que ceux qui n'en ont pas ou qui en ont un seul. À leur tour, les femmes ayant de 3 à 5 enfants sont plus nombreuses à être multipratiquantes que celles qui en ont 1 ou 2. Autant de données attestant qu'on ne trouve pas de relation logique ou stable entre les formes de la pratique et le nombre d'enfants vivant au foyer ${ }^{7}$.

Prises une à une, les conditions d'existence créées par l'activité professionnelle ou la présence d'enfants ne paraissent expliquer que faiblement les différences qu'on observe entre les hommes et les femmes au « centre de la vie». C'est certainement le cumul et la combinaison de ces « temps/préoccupations » qu'il faut prendre en compte pour comprendre pourquoi les femmes vont s'adonner à une seule activité physique ou sportive et peut-être renoncer à d'autres. Mais on doit aussi compter avec ce rapport, plus ou moins aisé et plus ou moins culpabilisé, que les femmes entretiennent avec le temps qu'elles sont susceptibles de prendre pour elles...

Chez les plus jeunes et les plus âgés, l'âge (qui renvoie à la fois à une condition physique et à une position de génération) atténue les différences de sexe. Au centre de la vie, en revanche, où se cumulent les temps et activités (travail professionnel, enfants, tâches domestiques, loisirs... ), où il faut combiner temps contraint et temps libre, problème au moins autant culturel qu'« arithmétique », ces différences auraient plutôt tendance à s'accentuer.

La position socioprofessionnelle infère à son tour d'inégales probabilités d'être mono ou multipratiquant (Tableau 2).

D'une manière générale, la multipratique est la plus fréquente parmi les cadres et professions intellectuelles supérieures et les professions intermédiaires ; autrement dit, dans les milieux sociaux les mieux dotés à la fois culturellement et financièrement et qui disposent, en outre, assez souvent d'une bonne latitude dans la gestion de leur temps travaillé (Irlinger et al., 1988). Les artisans, commerçants et chefs d'entreprise, les ouvriers et les employés sont également fréquemment multipratiquants (les 3 / 4 sont dans ce cas). Les agriculteurs occupent ici une position particulière : la moitié d'entre eux « seulement " associent au moins deux activités. Mentionnons que la taille de l'agglomération dans laquelle on réside introduit une différenciation superposable à la différenciation sociale : $32 \%$ des habitants des communes rurales (moins de 2000 habitants) sont monopratiquants alors que c'est le cas de $17 \%$ à $23 \%$ des autres Français, Parisiens compris. Si les agriculteurs sont davantage tournés vers la monopratique ${ }^{8}$, c'est certainement moins du fait de l'éloignement de la ville et de ses équipements ou structures d'accueil que du rapport très spécifique qu'ils entretiennent avec le temps, l'espace, les tâches à effectuer. Les saisons et les soins aux animaux scandent l'organisation des temps de la vie, de même les espaces naturels sont-ils des lieux de travail avant d'être des espaces de loisir...

Ainsi qu'on l'observe dans toutes les formes de pratiques, y compris sportives, l'appartenance sociale accentue les différences dues à l'appartenance de sexe (Tableau 2). Comme on le sait, il y a plus de distance entre catégories sociales quand on est une femme que lorsqu'on est un homme (Davisse \& Louveau, 1991) ; à l'exception des agriculteurs, la multipratique est à peu près également répandue parmi les hommes actifs. Pour les femmes, en revanche, celles qui sont cadres, membres des professions intermédiaires ou employées sont multipratiquantes dans des proportions comparables et bien plus fréquemment que les femmes ouvrières, les agricultrices et celles qui exercent une profession indépendante dans l'artisanat, le commerce ou en tant que chefs d'entreprise. Dans ce second ensemble de groupes sociaux, les différences entre hommes et femmes sont maximales : des ouvriers aux ouvrières, comme des indépendants (artisans, commerçants ou chefs d'entreprise) à leurs homologues féminines, la différenciation sexuelle des activités, espaces, temps... est des plus accusées. 


\section{Nature et degré de la multipratique}

La multipratique revêt concrètement des formes diverses : on peut la caractériser à la fois par des modalités de pratique dans le temps et l'espace ainsi que par le nombre d'activités ou de disciplines. Ce qu'on nomme, souvent pour aller vite, l'intensité de la pratique ou sa fréquence, et plus généralement l'investissement quantitatif dans la pratique prend, avec ces deux indicateurs, un sens aussi précis que complexe.

\section{Les secteurs spatio-temporels de la pratique}

Dans la vie quotidienne des individus, la pratique sportive peut se dérouler durant l'année (à l'intérieur ou à l'extérieur du domicile), elle peut se poursuivre également durant les vacances ou n'avoir lieu qu'à ce moment. Cet éventail de possibilités engendre d'éventuels cumuls ou combinaisons : on peut, par exemple, pratiquer à la fois à son domicile et en dehors de celui-ci ou encore à son domicile et durant les vacances seulement...

La majorité des pratiquants exercent une APS tout au long de l'année, y compris pendant les vacances ( $55 \%$ sont dans ce cas et $28 \%$ combinent plusieurs APS dans ces deux secteurs). Mais en fonction de leurs caractéristiques socio-démographiques, les Français se distribuent inégalement dans les différentes modalités de pratique renvoyant aux rapports entretenus avec les espaces / temps / activités (Tableau 3).

Sur le plan du sexe et alors qu'hommes et femmes sont à peu près également mono et multipratiquants, un discret partage s'opère principalement entre la pratique au domicile", plus " féminine », et celle s'effectuant hors du domicile, plus « masculine ».

Les femmes sont ainsi deux fois plus nombreuses que les hommes à pratiquer seulement à leur domicile ( $7 \%$ des femmes, contre 3,3\% des hommes sont dans ce cas).

Multipratiquantes, elles associent fréquemment une APS au domicile aux autres modalités de pratique (30\% des femmes sont dans ce cas contre $22,4 \%$ des hommes). En revanche, elles s'adonnent un peu moins souvent qu'eux à une APS se déroulant hors du domicile : associée ou non à d'autres secteurs, cette forme de pratique est exercée par 80,3\% des hommes et par 73,4 \% des femmes ${ }^{10}$. Exercer une APS hors de chez soi est a fortiori plus fréquent parmi les hommes que parmi les femmes quand cela concerne plusieurs activités. Ces constats renvoient à la question déjà mentionnée des rapports aux temps et aux espaces, aussi indissociables que gérés différemment des hommes aux femmes : ceux-ci investissent plus volontiers l'espace du dehors (ou hors du foyer), comme ils superposent mieux des pratiques sportives au temps de l'activité professionnelle. Les femmes, pour leur part, procèdent à l'inverse, optant plus volontiers pour les activités au domicile et aussi pour celles des vacances. Tout se passe comme si les femmes s'orientaient plutôt vers ce qui prend le moins sur le temps dévolu aux autres, comportement qui se nuance probablement selon les milieux sociaux, géographiques, l'âge...

Sur le plan de l'âge, on sait que la multipratique diminue au fil de la vie (Long \& Wimbush, 1979, 1985). Jusqu'à 49 ans, la forme modale de pratique reste inchangée : elle consiste à exercer une ou plusieurs APS en dehors du domicile durant l'année et durant les vacances. Par la suite, le nombre d'activités hors du domicile durant l'année tend à se réduire : alors qu'environ $40 \%$ des 12-49 ans pratiquent au moins 2 activités hors du domicile, $20 \%$ des personnes de plus de 50 ans sont dans ce cas. À partir de 65 ans, la pratique modale consiste à pratiquer une seule APS hors du domicile. Ainsi que le met en évidence la figure 1 , un passage s'opère à un moment de la vie, autour de la cinquantaine, entre une multipratique intense et la monopratique, modification attribuable, entre autres ${ }^{11}$, à une modification des capacités physiques.

L' appartenance socioprofessionnelle infère des modalités de pratique sensiblement distinctes ; on peut en rendre compte à trois niveaux :

Premièrement, la pratique des agriculteurs se distingue nettement de celle des autres 
catégories. Les hommes sont ici plus fréquemment monopratiquants que les autres et dans un tiers des cas, ils exercent une seule APS hors du domicile. Comme le montre le tableau 4, ce sont eux qui pratiquent le plus rarement plusieurs APS hors du domicile (associées ou non à un autre secteur de pratique).

Les agriculteurs sont également ceux qui pratiquent le moins souvent du sport en vacances, de même partent-ils beaucoup moins en vacances que les autres actifs ${ }^{12}$. Or, ainsi que le montre la figure 2 , les taux de départ en vacances et ceux de pratique d'une APS durant cette période covarient très nettement. Partir en vacances signifie se déplacer vers des lieux offrant des possibilités d'activités plus diversifiées ou plus originales, lieux qui seront choisis par certains aux fins mêmes d'exercer un sport particulier.

Deuxièmement, on observe de fortes différences des hommes aux femmes dans certains groupes sociaux. Il en est ainsi des artisans : pour eux, la modalité de pratique la plus fréquente consiste à associer une activité hors du domicile à une ou plusieurs durant les vacances. Les femmes (dont l'effectif est faible), pratiquent plus rarement que leurs congénères et que les autres femmes actives des APS en vacances, mais plus fréquemment que ces dernières une pratique au foyer.

Comme pour les artisans, les modalités de pratique des ouvriers diffèrent selon le sexe. Plus multipratiquants que les femmes, les hommes ne paraissent pas engagés dans une modalité de pratique dominante. Les ouvrières, en revanche, pratiquent surtout une seule APS durant les vacances. Pour elles, l'exercice de plusieurs sports hors du domicile durant l'année, c'est-à-dire au moment où il faut combiner les activités et les temps professionnel, domestique, familial... ${ }^{13}$, est nettement moins fréquent que pour les autres femmes, y compris les inactives sur le plan professionnel (Tableau 4).

Troisièmement, les cadres et professions intellectuelles supérieures ainsi que les membres des professions intermédiaires se trouvent, conformément aux attentes, dans des situations distinctes sinon opposées aux précédentes. Les hommes associent fréquemment une ou plusieurs APS durant l'année et en vacances. Ils sont également nombreux à exercer des APS dans les trois secteurs de pratique. Les modalités de pratique des femmes cadres - sur ce point, elles ne cessent de se différencier des autres femmes — sont similaires à celles de leurs homologues masculins.

\section{Le nombre d'activités pratiquées}

Le nombre d'activités pratiquées constitue un autre indicateur de la multipratique variant selon les caractéristiques socio-démographiques des individus. Peut-être même est-ce à ce niveau que les différences sont les plus accentuées, autrement dit, dès qu'il ne s'agit plus seulement de combiner des temps, mais d'avoir la possibilité ou la capacité de cumuler des activités - ce qui renvoie aux aspects relatifs au temps lui-même, mais aussi à l'argent et plus généralement à toutes les formes de capital qu'on peut y consacrer.

Si on considère les activités pratiquées sur toute l'année, vacances incluses (Tableau 5), les situations « extrêmes » semblent témoigner des différences sur le plan de l'âge et de l'appartenance sociale ; en revanche, les différences entre les hommes et les femmes restent étonnamment faibles :

- Pratiquer une seule activité est particulièrement le fait des plus âgés ainsi que des agriculteurs (respectivement $51 \%$ et $46 \%$ sont concernés par opposition à $25 \%$ de Français en moyenne);

Pratiquer 5 activités ou plus, en revanche, est très spécifiquement le fait des moins de 25 ans ( $25 \%$ en moyenne), des cadres et membres des professions intellectuelles supérieures (21 $\%)$ et, dans une moindre mesure, des professions intermédiaires (16\%). Moins de $5 \%$ des plus de 50 ans, des agriculteurs, des ouvriers et des professions indépendantes sont dans ce cas.

Cette dimension de la multipratique fait très clairement ressortir les différences entre groupes sociaux. 
En outre, apparaît, chez les cadres et professions intellectuelles supérieures, une différence entre hommes et femmes qu'on soulignera d'autant plus qu'elle se manifeste dans une catégorie où les deux sexes sont précisément les plus proches dans nombre de pratiques et tâches. Toutefois, si la multiplicité des activités finit par diviser les deux sexes, elle intervient lorsqu'il s'agit de pratiquer plus de 4 activités différentes dans l'ensemble de l'année ${ }^{14}$ !

Trente-six pour cent (36\%) des pratiquants s'adonnent à au moins deux disciplines durant l'année, $51 \%$ sont dans le même cas durant la période des vacances. Ce moment correspond donc à une ouverture des possibilités pour nombre d'individus, élargissement qui profite pratiquement à tous ; les vacances atténuent, en outre, les différences de sexe, de même que certaines différences liées à l'âge observées durant le reste de l'année (Tableaux 6 et 7). Pour leur part, les plus jeunes « ouvrent » très sensiblement leur éventail d'activités au moment des congés : $37 \%$ des 12-17 ans pratiquent 2 activités ou davantage durant l'année et $67 \%$ d'entre eux connaissent cette même situation durant les vacances !

Sur le plan des groupes sociaux, les vacances profitent aux cadres, aux membres des professions intermédiaires et aux employés : il sont alors nettement plus nombreux à opter pour plus de deux disciplines. En revanche, la pratique des artisans, commerçants, chefs d'entreprise ainsi que celle des ouvriers ne bénéficie à peu près pas de cette période de congés... quand les agriculteurs pour leur part s'avèrent globalement «perdants ». Du point de vue de la multipratique, la période des vacances creuse les écarts entre les groupes sociaux, à la mesure même des départs en vacances (Figure 2), c'est-à-dire de l'inégale probabilité qu'ont les individus de se déplacer vers des lieux se prêtant objectivement mieux à la pratique d'une APS.

\section{Des activités douces à la compétition : les variations des modalités de la multipratique}

\section{Les niveaux de pratique comme outil d'analyse}

Les pratiques d'agrément et d'hygiène d'une part et celles de compétition d'autre part constituent les deux modalités extrêmes de la variable de niveau de pratique qui sera utilisée comme principal instrument d'analyse de la multipratique dans cette partie.

Cette variable comporte quatre niveaux :

- niveau 1 : les pratiques d'agrément et d'hygiène. Activités d'entretien physique, de forme ou de loisir, ces pratiques ne comportent pas de finalités proprement sportives. La baignade, la promenade, la relaxation, l'utilisation du vélo d'appartement sont des exemples d'activités qui ont été incluses dans ce niveau. Lors de l'enquête, elles se déclarent sous des terminologies qui n'appartiennent généralement pas aux répertoires officiels des disciplines sportives $^{15}$.

- niveau 2 : les pratiques sportives non licenciées. Elles se déclarent à l'aide du répertoire sportif consacré ; on fait du vélo, on joue au tennis, etc., mais on ne possède pas de licence fédérale.

- niveau 3 : les pratiques sportives licenciées. Elles se distinguent des précédentes par leur inscription dans l'institution sportive, la licence témoignant de l'appartenance à un club affilié à une fédération sportive.

- niveau 4: les pratiques sportives compétitives. Il s'agit de pratiques licenciées qui comportent en plus une participation systématique ou ponctuelle aux compétitions officielles.

Un même sportif peut évidemment pratiquer des activités relevant de plusieurs niveaux de cette variable. Dans ce cas, c'est son activité la plus «sportive » qui classe le pratiquant. Ainsi, un licenciécompétiteur sera répertorié en tant que tel, indépendamment de ses éventuelles autres activités relevant des niveaux 1, 2 ou 3 de la variable. 


\section{Une hypothèse de bon sens contredite par les observations}

La multipratique peut être pensée, a priori, comme une mobilité, une dispersion, voire comme une inconstance sportive. Ainsi conçue, elle s'oppose à une stabilité, à une fidélité disciplinaire, garantes d'approfondissement, de compétence et de spécialisation. C'est cette deuxième manière d'être sportif qui semblait devoir s'accompagner, plus fréquemment que la première, de la prise de licence et, a fortiori, de la participation aux compétitions.

Cette hypothèse, qui relève du bon sens, d'une logique générale, ne se vérifie pas dans le champ des pratiques physiques et sportives. Elle se trouve, bien au contraire, invalidée de manière évidente par les données de l'enquête sur les pratiques sportives des Français.

La proportion de multipratiquants s'établit en effet à:

- $\quad 25,3 \%$ chez ceux qui ne pratiquent que des activités d'hygiène et d'agrément

- $\quad 83,6 \%$ chez ceux qui pratiquent du sport sans licence

- $\quad 93,2 \%$ chez les sportifs licenciés, qu'ils pratiquent ou non la compétition

Ainsi lorsque la pratique physique ne vise que le bien-être et ne puise pas dans le répertoire des disciplines proprement sportives, elle comporte une seule activité dans trois cas sur quatre. Dès que la pratique revêt un caractère proprement sportif, la pluralité des disciplines devient la règle, a fortiori chez les sportifs licenciés.

Par ailleurs, les sportifs licenciés, qu'ils soient ou non compétiteurs, sont en proportion beaucoup plus nombreux que les autres pratiquants à se livrer à des activités sportives aussi bien pendant les vacances que durant le reste de l'année, qu'il s'agisse d'exercices accomplis au domicile ou plus classiquement de sports pratiqués hors du foyer. C'est du reste au chapitre de ces dernières pratiques, les APS hors vacances et hors du domicile, que les groupes de niveau se différencient le plus clairement. Déclarent en effet plusieurs activités de ce type : 4,3\% des pratiquants d'hygiène et d'agrément, $31,6 \%$ des sportifs non licenciés et $61 \%$ des sportifs licenciés.

Quant à la monopratique, lorsqu'elle est le fait des adeptes d'activités d'hygiène et d'agrément, elle consiste le plus souvent en exercices accomplis chez soi (alors que cette catégorie d'activités comporte des pratiques hors du domicile telles que la baignade, la marche promenade, etc. ). Tout au contraire, la monopratique des adeptes d'activités sportives se situe plutôt hors du domicile et en dehors des vacances ; elle ne se présente que sous cette seule forme chez les rares compétiteurs monopratiquants ( $7,1 \%$ des compétiteurs sont monopratiquants).

\section{Niveaux de pratique et degrés de multipratique}

L'élévation du niveau de pratique s'accompagne d'un élargissement de l'éventail d'activités (Figure 3). Cette liaison apparait clairement dans le nombre moyen d'activités pratiquées dans chaque catégorie; cette moyenne est de :

- 1,2 pour les pratiquants d'hygiène et d'agrément,

2,8 pour les sportifs non licenciés,

et de 3,7 pour les sportifs licenciés.

Quant au nombre maximal d'activités pratiquées, il est de neuf pour les sportifs non licenciés tout comme pour les sportifs licenciés ; $0,5 \%$ des premiers et $2,4 \%$ des seconds en déclarent huit ou neuf ${ }^{16}$. Aucun pratiquant d'activités d'hygiène ou d'agrément de notre échantillon n'en pratique plus de trois.

\section{La multipratique des compétiteurs}

La distinction parmi les licenciés entre ceux qui font de la compétition (« compétiteurs ») et ceux qui n'en font pas («licenciés ») permet une analyse encore plus fine de la pratique multiple. 
La multipratique des seconds se constitue, bien plus souvent que celle des compétiteurs, grâce aux APS de vacances : $33,1 \%$ d'entre eux n'apparaîtraient pas dans la catégorie des multipratiquants sans leurs pratiques de vacances - ce n'est le cas que pour $20,2 \%$ des compétiteurs.

La multipratique des compétiteurs comporte en moyenne 3,8 activités contre 3,5 pour les licenciés. Mais ce qui se présente comme une différence faible sur les moyennes recouvre certaines disparités partielles assez fortes. Ainsi, la proportion des sportifs pratiquant plus de trois activités se situe à $45 \%$ chez les licenciés et à $54 \%$ chez les compétiteurs et pour plus de six activités respectivement à 11,9 et $17,1 \%$. On constate par ailleurs que près d'un compétiteur sur deux $(45,9 \%)$ s'adonne à des compétitions officielles ou amicales dans plusieurs sports.

La multipratique des compétiteurs n'est pas le seul fait des hommes ; à ce niveau de pratique, elle caractérise les habitudes sportives des deux sexes. Il est certes établi que de manière générale, les femmes témoignent d'une moindre appétence pour la compétition et qu'elles s'y engagent effectivement en de moindres proportions (sur 100 compétiteurs, 75 sont des hommes et 25 des femmes); mais celles qui s'y adonnent jouent pleinement le jeu, elles déploient une sportivité en tous points comparable à celle de leurs homologues masculins, comme si à partir de ce seuil, le sport prenait le pas sur le sexe.

L'analyse des données montre donc que l'hypothèse posée précédemment d'une opposition entre le niveau de pratique et la multipratique doit être rejetée. Des activités physiques d'agrément et d'hygiène jusqu'à la compétition sportive officielle, la proportion des multipratiquants, tout comme le nombre d'APS pratiquées, augmentent de manière significative.

Cependant un dernier doute subsiste : la compétition officielle comporte elle-même différents niveaux. Aux plus élevés, on imagine mal que la spécialisation qui nécessite des temps d'entrainement dépassant généralement vingt heures par semaine, sans compter les stages et les déplacements en France ou à l'étranger pour participer aux compétitions, puisse se faire autrement qu'au détriment d'autres activités et en particulier du nombre d'APS pratiquées. Or, même pour ces compétiteurs de haut niveau, les données observées s'inscrivent en faux au regard de l'hypothèse initiale.

Dans l'enquête sur les pratiques sportives des Français, nous avons distingué cinq niveaux de compétition : amical ou local, départemental, régional, national et international. Dans le traitement des données effectué pour ce travail, nous avons exclu le premier niveau du champ de la compétition officielle et avons regroupé, pour des raisons évidentes d'effectifs, le niveau national et le niveau international.

Les chiffres en gras du tableau 8 signalent, pour chaque degré de multipratique, le niveau de compétition comportant la plus forte proportion de compétiteurs. La disposition en diagonale de ces chiffres permet de visualiser la covariation entre degré de multipratique et niveau de compétition. Cette covariation se trouve confirmée par le calcul du nombre moyen d'activités pratiquées : ce dernier est de 3,5 chez les sportifs faisant des compétitions départementales, 3,9 chez ceux opérant au niveau régional et 4,1 au niveau national ou international.

Compte tenu des exigences, notamment temporelles, de la compétition sportive, il est évident que dans l'éventail des activités déclarées par les compétiteurs, une seule est dans la grande majorité des cas pratiquée à un niveau élevé. Les autres APS ont pour ces sportifs un statut d'activités de loisir, certaines d'entre elles pouvant cependant être choisies comme adjuvants à l'entraînement dans la discipline sportive principale. Cette interprétation de bon sens s'appuie notamment sur le nombre moyen de licences détenues qui n'est guère plus élevé chez les compétiteurs de niveau national ou international $(1,31)$ que chez l'ensemble des licenciés sportifs où il se situe à 1,26 .

L'exploitation des données recueillies par l'enquête sur les pratiques sportives des Français permet donc de rejeter l'hypothèse d'une opposition, d'une concurrence, entre le nombre d'activités pratiquées et le niveau de pratique atteint. Tout à l'opposé, on constate que le nombre d'activités pratiquées augmente parallèlement au niveau de pratique. Même à un haut niveau de compétition, les investissements nécessaires à la spécialisation dans un sport particulier n'excluent pas, bien au contraire, l'ouverture 
large de l'éventail des APS pratiquées comme activités complémentaires ou plus généralement de loisir.

On retrouve ici un constat déjà fait dans un travail antérieur portant sur les rapports entre la pratique sportive et la consommation de sport télévisé (Irlinger, 1994): les différentes pratiques d'exercice, de communication et de consommation relevant du champ sportif tendent à s'associer, à se cumuler plutôt qu'à se concurrencer, ne serait-ce que sur le plan du temps investi.

L'opposition qui semblait aller de soi entre spécialisation et multipratique sportive n'est pas nouvelle. Historiquement, elle a constitué dans les années vingt, l'axe majeur de l'opposition entre les théoriciens du sport et ceux d'une éducation physique généraliste. Ces derniers (Hébert, 1925) reprochaient au sport et à la spécialisation sportive sa pauvreté motrice et sa stéréotypie gestuelle qu'ils pensaient plus ou moins consciemment à travers le modèle du travail industriel à la chaîne. Dans les années soixante et soixante-dix, ce débat, nourri des mêmes préoccupations pédagogiques et humanistes, redevint d'actualité dans la lutte menée par des enseignants d'éducation physique contre la spécialisation sportive précoce mise en oeuvre par des entraîneurs qui y voyaient la condition incontournable pour obtenir des résultats sportifs de valeur internationale.

L' analyse des données de l'enquête sur les pratiques sportives des Français montre que spécialisation sportive et multipratique ne constituent pas les deux termes d'une alternative, mais se conjuguent bien au contraire dans la réalité de la pratique sociale. Le caractère idéologique de cette opposition, révélée par l'approche historique, se trouve ainsi empiriquement confirmé.

\section{Conclusion}

Appréhendée à travers différents indicateurs qui se combinent (s'adonner à un nombre plus ou moins grand d'activités, soit dans un seul secteur, soit, cas le plus fréquent, à la fois durant les vacances et durant l'année « active », dans et/ou hors son domicile), la multipratique est la façon de «bouger» la plus répandue au sein de la population française. Le fait d'être un homme, et encore d'appartenir à la catégorie sociale des cadres et professions intellectuelles supérieures, rend plus probable cette forme de sportivité, le fait d'avancer en âge la rendant en revanche moins fréquente. La prise en compte du nombre d'activités accentue ces différenciations liées aux appartenances d'âge ou de groupe social. La période des vacances est certainement propice à une plus intense activité pour tous, mais ce moment n'induit pas une égalité sur ce point ; ceux qui partent en vacances demeurent plus favorisés que les autres.

Engagement intense et assidu dans une discipline et multipratique ne sont pas antinomiques, c'est le second aspect qui ressort nettement de l'analyse. Les licenciés compétiteurs, autrement dit les pratiquants qui s'adonnent à la pratique la plus instituée, la plus contraignante aussi sur le plan temporel et organisationnel, sont aussi ceux qui ont choisi le plus fréquemment de pratiquer plusieurs activités sans interruption sur l'année ; mieux, ces sportifs s'adonnent à plus de disciplines différentes que les autres. Le fait qu'ils se situent aux plus hauts niveaux de la compétition ne représente pas un frein, bien au contraire. L'hypothèse de bon sens énonçant que la spécialisation va de pair avec une focalisation, voire une fixation exclusive sur un sport, s'avère donc pour le moins invalidée.

Depuis l'enquête INSEE sur «Les comportements de loisirs des Français » de 1967, qui a livré les premières données quantitatives sur les pratiques sportives en France, on sait que ces dernières se sont massifiées, qu'elles ont concerné une part sans cesse croissante de la population. Ce phénomène participait d'une expansion quantitative des activités de loisir, elle-même corrélative de l'augmentation du « temps libre ». Mais on connaît aussi la manière inégale dont ces pratiques se distribuent dans les différents groupes sociaux ${ }^{17}$, fait durable bien qu'évolutif à son tour. Cette différenciation sociale par les APS s'opère essentiellement aujourd'hui sur un plan qualitatif : elle se trouve accentuée si, au lieu de prendre en considération le seul fait de pratiquer, on ne retient que la multipratique.

L'étude attentive des différentes formes que revêt cette multipratique rend particulièrement visible ce que les pratiques sportives sollicitent, à l'instar d'ailleurs des autres pratiques culturelles ou de loisir : le 
capital dont on dispose et qu'on peut ou qu'on va investir dans ces activités. Qu'il s'agisse de loisir ou de compétition, il s'avère que les « nantis cumulent» pour le dire en termes prosaïques.

Majoritairement combinées ou cumulées aux différents temps de l'année, les pratiques des cadres et professions intellectuelles supérieures mobilisent à l'évidence toutes les formes de capital que requièrent les APS et dont ces groupes sociaux sont très largement dotés, de manière précisément cumulative (sur les plans économique, scolaire, culturel, social... ). Leur « richesse », à la fois concrète et symbolique, les porte d'autant plus vers la diversité et la quantité d'activités qu'ils en ont " les moyens », leur attention soutenue pour leur apparence corporelle pouvant être ici surdéterminante. La «polyvalence » sportive suppose certaines conditions sociales de possibilité.

Les compétiteurs, à la fois spécialisés et multipratiquants, n'appartiennent pas tous à ces groupes sociaux. Mais on pourrait reprendre à leur propos le concept de richesse : les capacités physiques, les savoir-faire techniques et plus généralement la culture physique dont on dispose ne constituent-ils pas un véritable capital corporel qu'on peut investir et encore transférer d'un sport à un autre ?

Reste enfin et pour tous la question du temps comme capital : pas d'équation possible entre la quantité de temps libre et le nombre de pratiques, entre la quantité de temps consacré à une activité et l'élimination de toute autre. La richesse, ici, est certainement cette possibilité, très inégalement distribuée, d'avoir prise sur la gestion de son temps : sous cette condition, tout se passe comme si l'on trouvait d'autant plus le temps de faire telle ou telle activité qu'on la perçoit comme souhaitable, autrement dit qu'elle répond à une désirabilité sociale.

\section{NOTES}

1. Pour une description plus précise de cette enquête, on se reportera à l'encadré placé en annexe. Pour connaître l'ensemble des résultats, voir le rapport de l'enquête : IRLINGER, P., LOUVEAU, C., METOUDI, M. (1988). Les pratiques sportives des Français ; enquête sur les usages sportifs du temps libéré. Étude commandée par le Secrétariat d'État à la Jeunesse et aux Sports, Paris : INSEP, 2 tomes, 735 pages.

2. Nous avons, lors de cette enquête, opté pour un questionnement le plus ouvert possible : nous souhaitions en effet recenser toutes les activités physiques et sportives, les plus traditionnelles (instituées, compétitives), aussi bien que les plus récentes (informelles, de loisir et d'entretien).

3. On observe à peine plus de monopratiquants parmi les femmes $(24,7 \%)$ que parmi les hommes $(20,2 \%)$.

4. Considéré comme un déterminant majeur susceptible de peser sur nombre de pratiques sociales, le temps doit être appréhendé dans ses multiples composantes, plus ou moins objectivables et toujours imbriquées : la dimension quantitative du temps est la plus visible, mais elle comprend, et dissimule parfois d'autres dimensions qualitatives. Il faut en effet considérer les façons différentes de vivre le temps, éminemment dépendantes de la subjectivité de chacun (avoir le sentiment de le perdre, d'en manquer, d'en prendre... ), qui sont loin de toujours coïncider avec les données quantitatives.

5. En 1987, l'âge moyen des mères à la naissance de leur premier enfant était de 26,4 ans si l'on considère à la fois les naissances légitimes et naturelles (voir Couet, C., Tamby, I. (1992). La situation démographique en 1992. Mouvement de la population. Paris : INSEE, coll. Démographie et Société, p. 92).

6. Pour les données concernant le mariage, les enfants et l'activité professionnelle des femmes, voir INSEE, Données sociales (1987, 1990 et 1993 en particulier).

7. L'enquête sur « Les pratiques sportives des Français », (op. cit.) avait, sur le plan général de la pratique, assez nettement invalidé cet élément comme " cause » de l'absence d'activité sportive par exemple ; l'allégation relève davantage de l'idée reçue que de l'explication sociologique.

8. Il faut en outre préciser que c'est parmi les agriculteurs qu'on trouve le plus grand nombre de nonpratiquants.

9. La pratique au domicile consiste en exercices physiques faits chez soi ; il s'agit le plus souvent d'exercices de culture physique : gymnastique, assouplissements, musculation, travail des abdominaux, utilisation d'un vélo d'intérieur ou d'une machine à ramer. 
10. Rappelons que tous les pourcentages concernant la pratique des APS sont calculés en utilisant comme base $(100 \%)$ la population des pratiquants.

11. Dans la recherche des causes, on ne peut exclure la position de génération que les individus occupent ; les personnes âgées de 50 ans ou plus au coeur des années 80 n'ont certainement pas eu, dans leur jeunesse en particulier, le même rapport aux APS que les jeunes d'aujourd'hui, à une époque où la pratique n'était pas un phénomène de masse.

12. Rappelons que $57,5 \%$ des Français partent en vacances sur une année, le taux de départ aux sports d'hiver étant constant depuis 1978, il concerne 7 à $8 \%$ des Français (INSEE, Données sociales, 1987).

13. Les dimensions culturelles du temps ont déjà été mentionnées ; on doit en outre considérer que les effets diviseurs (ou distinctifs) du temps et des revenus (entre les femmes et entre les groupes sociaux) tient aussi à d'inégales possibilités de s'adjoindre des aides domestiques (équipement ou personnes) et plus généralement de déléguer les tâches.

14. $5 \%$ des hommes cadres ou membres des professions intellectuelles supérieures sont dans ce cas pour $14 \%$ de leurs homologues féminines.

15. Pour une présentation plus précise et plus explicite des modalités de cette variable secondaire que nous avons baptisée « Nivospor ", on se reportera au rapport de recherche « Les pratiques sportives des Français », op. cit., pp. 52-55.

16. Cette pratique sportive intense peut se décliner sous des formes multiples. Nous l'illustrerons à travers deux exemples. Le premier concerne un homme âgé de 36 ans, marié, père de deux enfants et appartenant aux « cadres et professions intellectuelles supérieures ». Durant l'année, il joue au tennis deux à trois fois par semaine, il fait un jogging hebdomadaire, et du vélo et de la natation une fois par mois ; en outre, quatre à cinq fois par semaine, il fait à son domicile des exercices pour renforcer ses abdominaux. Ses vacances sont entièrement orientées vers la pratique sportive puisqu'il s'y adonne alors durant 40 heures par semaine et parfois plus ; l'hiver il fait du ski alpin, du ski de fond et du patinage, l'été, de la planche à voile et de la randonnée pédestre. Le second exemple se rapporte à un jeune homme de 17 ans, scolarisé, qui, durant l'année, fait du basket quatre à cinq fois par semaine (activité qui l'amène à participer à des compétitions), il fait également du judo et de la natation une fois par semaine et du tennis une à deux fois par mois. Durant ses vacances, il joue au football, aux boules, il court, il fait du ski et du vélo.

17. Des données nationales l'ont montré ; on peut par exemple citer des enquêtes telles celle de l'INSEE (Garrigues, 1988) ou du CREDOC (1994) ; sur l'analyse des processus, voir par exemple Pociello et al. (1981) et Clément, Defrance, Pociello (1994).

\section{BIBLIOGRAPHIE}

CIBOIS, P. (1984). L'analyse des données en sociologie. Paris : PUF, coll. « Le sociologue », 212p.

CLÉMENT, J.-P., DEFRANCE, J., POCIELlO, C. (1994). Sport et pouvoirs au XXème siècle. Grenoble : PUG, 204p.

CREDOC (1994). Le comportement des consommateurs d'articles de sport. Paris : CREDOC. (Rapport d'enquête réalisée sur un échantillon de 1010 personnes âgées de 14 à 65 ans).

DAVISSE, A., LOUVEAU, C. (1991). Sports, école, société : la part des femmes. Joinville-le-Pont : Actio, 288p.

GARRIGUES, P. (1988). Evolution de la pratique sportive des Français de 1967 à 1984. Paris : Les collections de l'INSEE, série $\mathrm{M}, \mathrm{n}^{\circ} 134$.

HÉBERT, G. (1925). Le sport contre l'éductaion physique. Paris : Librairie Vuibert, 135p.

INSEE (1987, 1990, 1993). Données sociales. Paris : INSEE. 
INSEE (1970). Les comportements de loisirs des Français. Paris : Les collections de l'INSEE, M2. (Echantillon de 6637 personnes âgées de 14 ans et plus).

INSEE (1989). Les pratiques de loisirs vingt ans après - 1967 / 1987-1988. Paris : Les collections de l'INSEE, série Résultats, $\mathrm{n}^{\circ} 13$.

INSEE (1989). Les pratiques de loisir. Enquête1987-1988. Paris : Les collections de l'INSEE, série Résultats, $\mathrm{n}^{\circ} 3$.

IRLINGER, P., LOUVEAU, C., METOUDI, M. (1988). Les pratiques sportives des Français. Paris : INSEP, 2 tomes, $735 \mathrm{p}$.

IRLINGER, P., LOUVEAU, C., METOUDI, M. (1992). " Sociologie des Activités Physiques et Sportives : promenade dans la littérature », Revue STAPS, 27 : 53-72.

IRLINGER, P. (1987). " Taux de pratique sportive, indicateur ou artefact ? », in Sport et changement social, Actes des premières journées d'études de la société française de sociologie du sport, 3 et 4 avril 1987, Bordeaux : Maison des Sciences de l'Homme d'Aquitaine, p.229-237.

IRLINGER, P. (1993). « La demande de sport en France, essai de détermination d'un profil de marché », in. LORET, A. (sous la direction de) Sport et management. Paris : Dunod, p.185-207.

IRLINGER, P. (1994). "The contribution of televised sports to the spread of sports activities", International Review for the Sociology of Sport, 29(2) : 201-210.

KAMPHORST, T.J., ROBERTS, R. (Eds.) (1989). Trends in sports. A multinational perspective. Pays Bas : Gioradano Bruno Culemborg, 422p.

LEROUX, P. (1970). Les comportements de loisirs des Français. Rapport de l'enquête INSEE de 1967. Paris : Les collections de l'INSEE, série M, no 2.

LOIRAND, G. (1989). Pratiques sportives et cycles de vie; influence des événements de la vie sur les carrières sportives. Mémoire pour le DEA STAPS "Pratiques, consommations et espaces sportifs », Université Paris-Sud - Orsay.

LONG, J., WIMBUSH, E. (1979). Leisure and the other-50s. Londres : SSRC / Sports council.

LONG, J., WIMBUSH, E. (1985). Continuity and change : Leisure around retirement. Londres : ESRC / Sports council.

LOUVEAU, C. (1995). «Sport masculin / sport féminin. Activité physique de loisir/ sport de compétition », in INSEE (ed.), Les Femmes, Paris : INSEE, coll. Contours et caractères, p.192-195.

POCIELLO, C. (sous la direction de) (1981). Sports et société. Approche socioculturelle des pratiques. Paris : Vigot, $377 \mathrm{p}$.

RAPOPORT, R., RAPOPORT, N. (1975). Leisure and the family life-cycle. Londres : Routledge.

ROBERTS, K., MINTEN, J.H., CHADWICK, C., LAMB, K.L., BRODIE, D.A. (1991). "Sporting lives : a case study of leisure careers", Loisir et société, 14 (1): 261-284.

RUDMAN, W.J. (1989). "Age and involvement in sport and physical activity", Sociology of Sport Journal, $6: 228-246$. 


\section{ANNEXE}

\section{L'ENQUÊTE SUR LES PRATIQUES SPORTIVES DES FRANÇAIS}

\section{Objectifs}

Une enquête focalisée sur les effets de la politique " sport pour tous » avait été demandée au Laboratoire de sociologie du sport de l'INSEP par le ministère de la Jeunesse et des Sports. Après consultation des principaux acteurs institutionnels du champ sportif, les sociologues de l'INSEP ont proposé puis réalisé une enquête plus large visant l'ensemble des pratiques physiques et sportives des Français. Les questions posées étaient sous-tendues par un système d'hypothèses portant particulièrement sur les transformations des pratiques (diversification, désinstitutionnalisation, retour de la dimension ludique. . .) et des représentations qui les génèrent (perte du monopole de la conception compétitive du sport).

\section{Échantillon}

3000 personnes représentatives de la population française de 12 à 74 ans.

Quotas : sexe-activité, âge et catégorie socioprofessionnelle de la personne de référence ; stratification par régions et tailles d'agglomération. Échantillon établi par l'Institut National d'Études Démographiques (INED).

\section{Financement}

Ministère de la Recherche et de la Technologie (Décision d'aide Nº 84 D 1581) Rémunération des chercheurs par le ministère de la Jeunesse et des Sports.

\section{TABLEAUX}

\section{TABLEAU 1}

Proportion de mono et de multipratiquants selon l'âge et le sexe

\begin{tabular}{lllllll}
\multirow{\text{Âge}}{*}{} & Hommes & \multicolumn{3}{c}{ Femmes } & \multicolumn{2}{l}{ Ensemble } \\
\cline { 2 - 7 } & Mono. & Multi. & Mono. & Multi. & Mono. & Multi. \\
\hline $12-17$ & 11 & 89 & 16 & 84 & 14 & 87 \\
$18-24$ ans & 19 & 81 & 21 & 80 & 20 & 80 \\
$25-34$ ans & 16 & 84 & 22 & 79 & 19 & 81 \\
$35-49$ ans & 17 & 83 & 29 & 72 & 22 & 78 \\
$50-64$ ans & 32 & 68 & 37 & 63 & 34 & 66 \\
$65-75$ ans & 48 & 52 & 51 & 49 & 49 & 51 \\
\hline
\end{tabular}




\section{TABLEAU 2}

Proportion de mono et de multipratiquants selon la CSP et le sexe

\begin{tabular}{lrlllll} 
CSP & \multicolumn{2}{l}{ Hommes } & \multicolumn{2}{l}{ Femmes } & \multicolumn{2}{l}{ Ensemble } \\
\hline \multicolumn{2}{l}{ Mono. } & Multi. & Mono. & Multi. & Mono. & Multi. \\
\hline Agriculteurs & 45 & 55 & 50 & 50 & 46 & 54 \\
Art., comm., & chefs15 & 85 & 42 & 58 & 24 & 76 \\
Cadres et prof. intell. sup. 15 & 85 & 11 & 89 & 13 & 87 \\
Professions & 14 & 86 & 24 & 76 & 19 & 81 \\
Employés & 19 & 81 & 26 & 74 & 24 & 76 \\
Ouvriers & 22 & 78 & 43 & 57 & 26 & 74 \\
Femmes au foyer & & & 32 & 68 & & \\
\hline
\end{tabular}




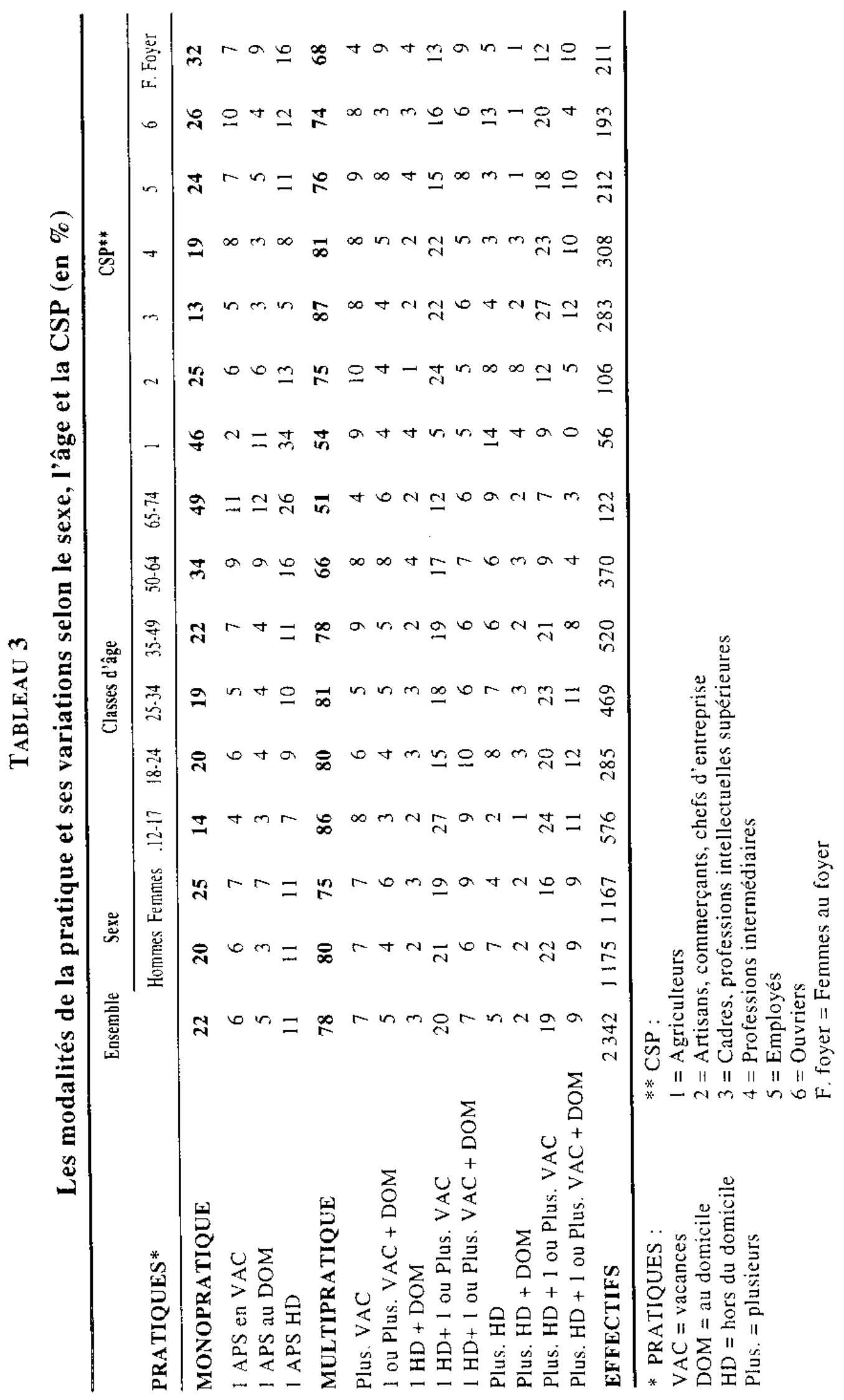




\section{TABLEAU 4}

Proportion des multipratiquants hors domicile selon la CSP et le sexe

\begin{tabular}{lll} 
CSP & Sexe & \\
\cline { 2 - 3 } & Hommes & Femmes \\
\hline Agriculteurs & 30 & - $^{*}$ \\
Art., comm., chefs entreprise & 36 & 24 \\
Cadres et prof. intell. sup. & 44 & 44 \\
Professions intermédiaires & 45 & 33 \\
Employés & 45 & 26 \\
Ouvriers & 42 & 19 \\
Femmes au foyer & & 29 \\
\hline
\end{tabular}

* L'effectif des agricultrices est trop faible : $n=12$. 


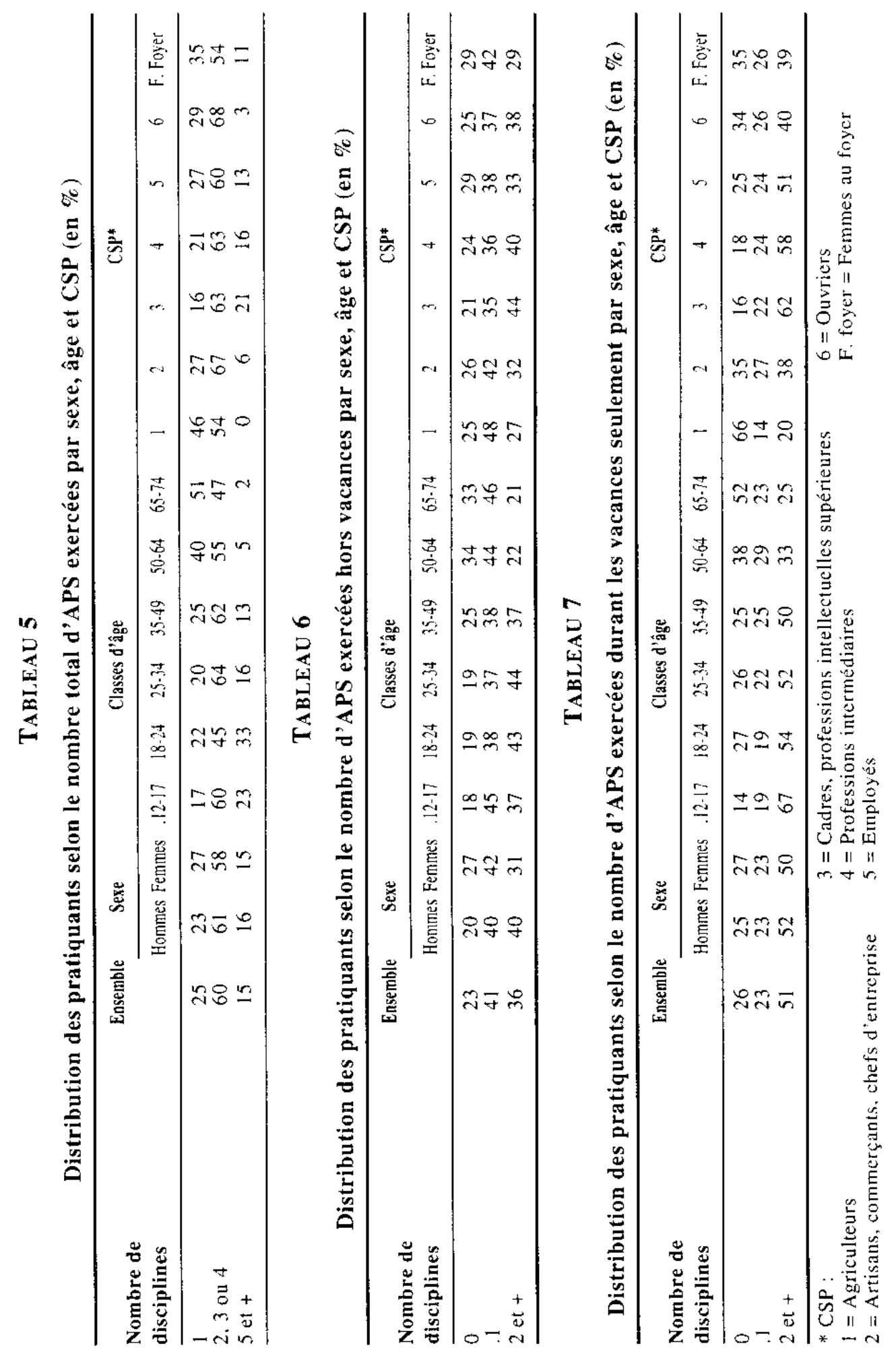




\section{TABLEAU 8}

Nombre d'APS pratiquées selon le niveau atteint en compétition officielle (en \%)

\begin{tabular}{|l|c|c|c|}
\hline \multirow{2}{*}{$\begin{array}{l}\text { Nombre total d'APS } \\
\text { pratiquées }\end{array}$} & \multicolumn{3}{|c|}{ Niveau de compétition } \\
\cline { 2 - 4 } & départemental & régional & $\begin{array}{c}\text { national ou } \\
\text { international }\end{array}$ \\
\hline 1 & & & 7,4 \\
\hline 2 & $\mathbf{1 0 , 6}$ & 8,3 & 13,8 \\
\hline 3 & $\mathbf{2 0 , 4}$ & 9,7 & 20,2 \\
\hline 4 & 22,5 & $\mathbf{2 3 , 4}$ & 17 \\
\hline 5 & 20,4 & $\mathbf{2 6 , 2}$ & $\mathbf{1 9 , 1}$ \\
\hline 6 à 9 & 14,1 & 13,8 & $\mathbf{2 2 , 3}$ \\
\hline
\end{tabular}

\section{FIGURES}

Figure 1 Distribution par âge des pratiquants selon le nombre des disciplines exercées

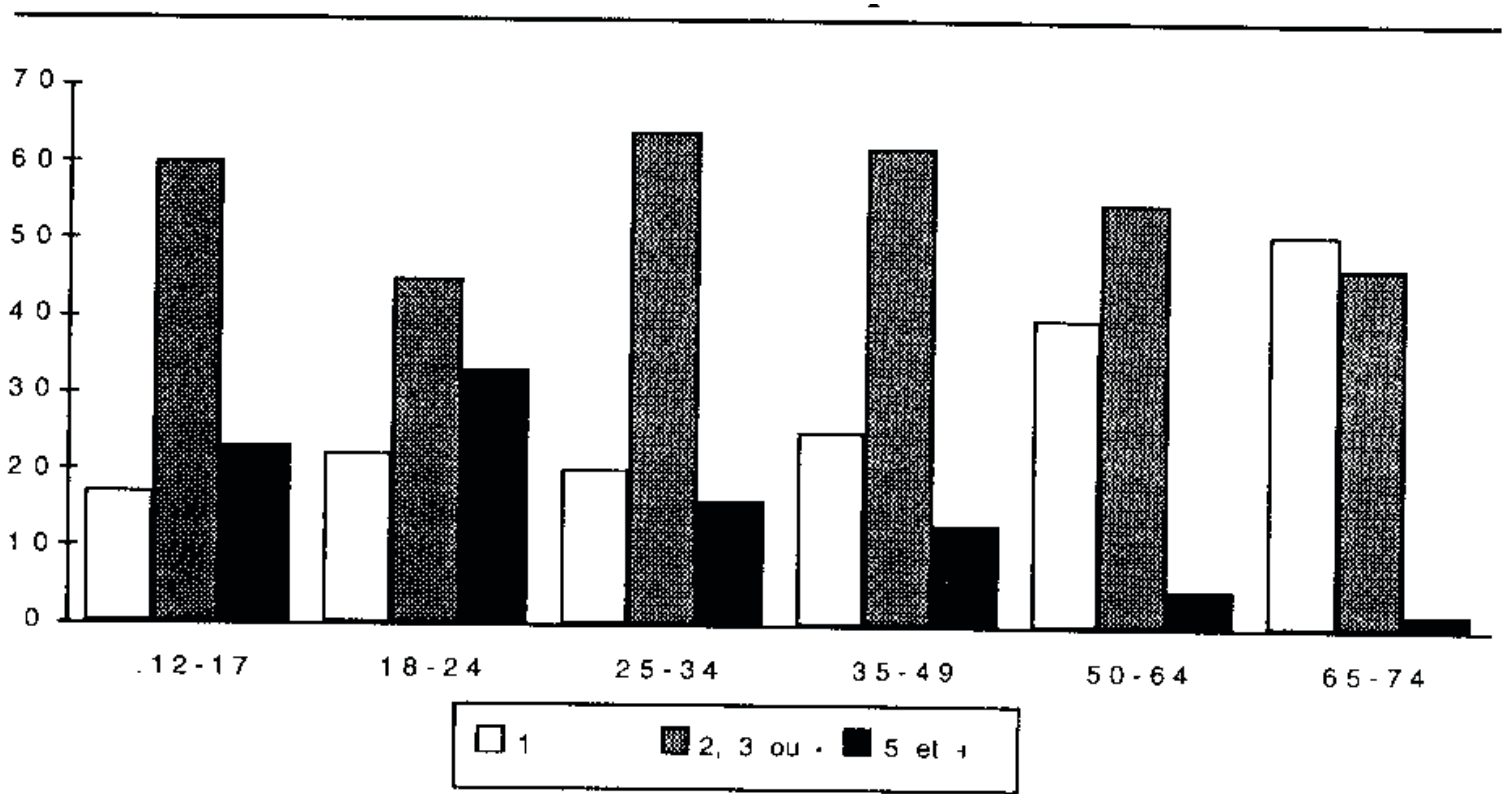


Figure 2 Proportions comparées des départs en vacances et des pratiquants d'APS en vacances selon la CSP

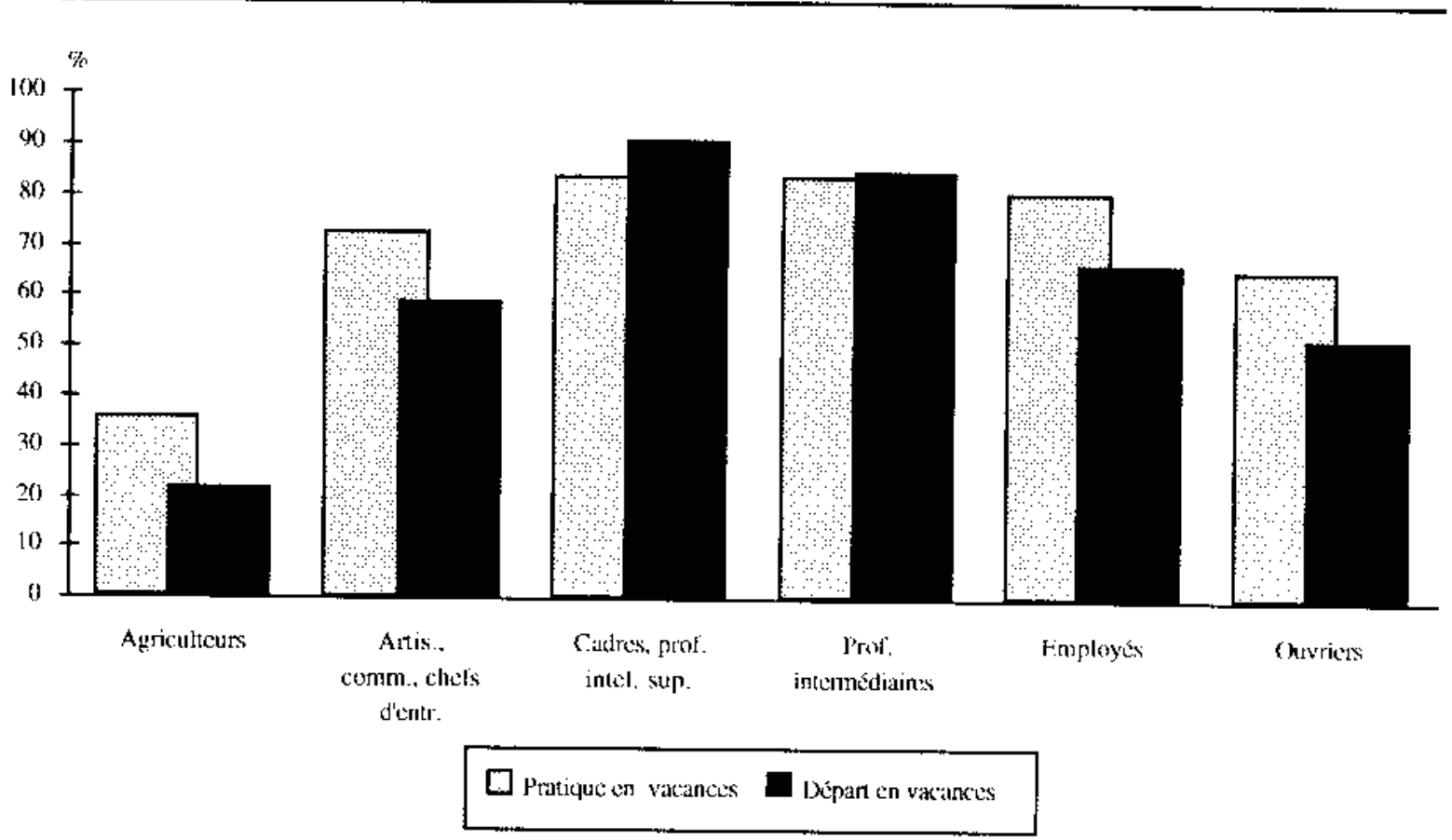

Figure 3 Distribution des pratiquants par niveau, selon le nombre d'activités

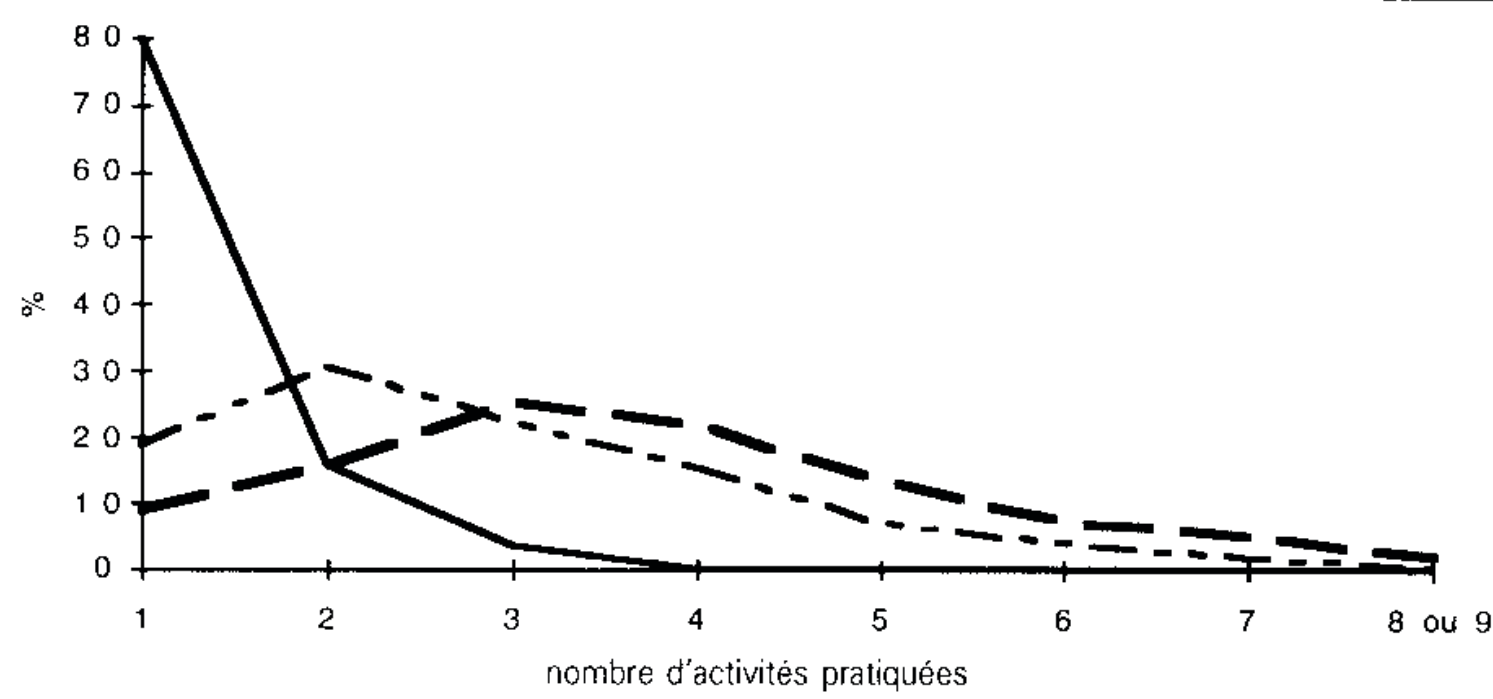

pratiquants d'hygiène - - - non licenciés et d'agrément

- licenciés pratiquant ou non la compétition 